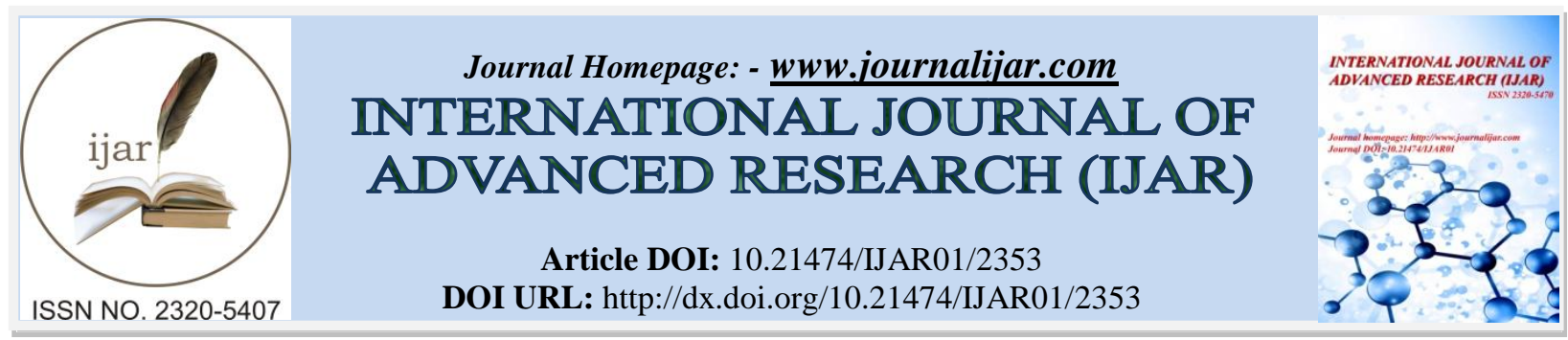

RESEARCH ARTICLE

\title{
COMPARISON OF THE REPRODUCIBILITY OF MEASUREMENTS OBTAINED BY A 1ST GENERATION, 2ND GENERATION AND 3RD GENERATION PERIODONTAL PROBE.
}

\author{
*Gulnar Dara Sethna ${ }^{1}$, Rajesh Prabhakar Gaikwad ${ }^{2}$, Akshaya Bhupesh Banodkar ${ }^{3}$, Nilofar Badshah \\ Attar $^{4}$, Chitra Laxmikant Patil ${ }^{5}$ \\ ${ }^{1}$ Assistant Professor, Department of Periodontology, Government Dental College and Hospital, Mumbai, India. \\ ${ }^{2}$ Associate Professor, Department of Periodontology, Government Dental College and Hospital, Mumbai, India. \\ ${ }^{3}$ Associate Professor, Department of Periodontology, Government Dental College and Hospital, Mumbai, India. \\ ${ }^{4}$ Assistant Professor, Department of Periodontology, Government Dental College and Hospital, Mumbai, India. \\ ${ }^{5}$ Dental surgeon, Department of Periodontology, Government Dental College and Hospital, Mumbai, India.
}

\section{Manuscript Info}

1.......................

Manuscript History

Received: 30 September 2016

Final Accepted: 30 October 2016

Published: November 2016

Key words:-

Florida probe, UNC 15 probe, PDT

Pressure sensitive probe, periodontal

disease ,Probing depth,Clinical

Attachment level.

\section{Abstract}

Background: The assessment of clinical attachment level (CAL) remains the gold standard for diagnosing and monitoring periodontal disease .There are certain errors inherent in the use of periodontal probes like probing force, visual errors in identifying the cementoenamel junction (CEJ), gingival inflammation and misrecording measurements. The main aim of our study is to compare the reproducibility of measurements obtained by the UNC-15 Periodontal probe (1st generation), PDT Pressure sensitive probe (2nd generation) and the Florida probe (3rd generation probe). The study was conducted by two trained examiners.

Methods: Thirty adult healthy subjects, with an age-range of 25-60 years, having moderate to advanced periodontitis in otherwise systemically healthy patients were included in this study . Probing depth (PD) and clinical attachment level (CAL) were recorded on three teeth in the same quadrant.Four measurements per tooth were recorded (mesio-buccal, mid-buccal, disto-buccal and mid-palatal/lingual.The measurements recorded by using three generations of probes were subjected to statistical analysis for comparison of accuracy and reproducibility.

Results: The PD and CAL measurements obtained by UNC 15 probe were higher than PDT pressure sensitive probe and Florida probe but there was no statistical significance in variability of PD and CAL measurements between the conventional and the controlled pressure probe. Inter -examiner consistency of Florida probe was found to be better than UNC-15 and PDT pressure sensitive probe.

Conclusion: Within the limitations of our study, Florida probe has proved to have the greatest potential for accuracy and reliability for measurements of CAL than PDT Pressure sensitive probe and manual probe (UNC-15).

Copy Right, IJAR, 2016,. All rights reserved. 


\section{Introduction:-}

A periodontal diagnosis is an important label that clinicians place on a patient's periodontal condition or disease. The periodontal pocket is the cardinal symptom of periodontitis and periodontal probe remains the best clinical diagnostic tool for the collection of information regarding the health status and the attachment level of periodontal tissues (Arthur F.H 1997). Clinical probing is one procedure that has been used extensively to assess changes in periodontal attachment over time (Preshaw et al.,1999). Factors influencing the probing depth measurement of periodontal pocket includes gingival health and disease, dental prosthesis, probing force and examiner variability, as well as the probing design (Andrade et al., 2012). Increased precision of periodontal probing is of clinical importance because the reported prevalence of disease activity as identified by clinical attachment level (CAL) change clearly depends on the threshold used for identifying whether the loss of attachment has occurred (Jeffcoat 1992 ) Pocket depth is less important than the level of attachment because it is not necessarily related to bone loss (Newman et al., 2006).

To date, the periodontal probe is the only instrument that has been found to be reliable and convenient in pocket examination. Philstrom in 1992 had classified these periodontal probes as first, second and third generation periodontal probes. The first generations are conventionally thin, with millimetre marked at selected points; the second generation being introduced to provide a constant probing force; and the third generation is the automated probe which has been introduced, following an NIDR workshop, to further reduce measurement errors (Parakkal ,1979)

\section{UNC 15 Probe:-}

Today, the University of North Carolina probe (PCP UNC 15, Hu Friedy Manufacturing Co., Chicago, IL, USA), with colour coding of every millimetre demarcation, is probably the preferred instrument in clinical research if conventional probes are required (Arthur 1997). A series of investigations that identified a positive correlation between probing force and depth of probe penetration led to the construction of probes with constant probing force.Probing forces can vary from $20 \mathrm{gm}$ to as much as $400 \mathrm{gm}$ when using conventional, rigid, non-pressuresensitive periodontal probe (Hunter et al.,1990)

\section{PDT pressure sensitive probe:-}

PDT Pressure sensitive probe makes it possible to maintain consistency of probing force between clinicians and from examination to examination. Black markings on the white thermoplastic PDT Sensor Probes are clearly defined providing a high contrast to oral tissue making measurements easy to read. PDT Sensor Probe is virtually non-abrasive in use and cannot mark the surface of any restoration.The flexible plastic probe tip could be exerted with a pressure of 15 grams during probing. Pressure-indicating marks are present on the PDT pressure sensitive probe. When the operator force reached 15 grams, the shank moved up to match the mark.

\section{Florida probe:-}

The Florida Probe (Florida Probe Corp, Gainesville, FL) was devised by Gibbs et al in 1988 consisting of a probe hand piece and sleeve, a displacement transducer, a foot switch, and a computer interface/personal computer. The hemispheric probe tip has a diameter of $0.45 \mathrm{~mm}$ and the sleeve has a diameter of $0.97 \mathrm{~mm}$.Constant probing pressure of $15 \mathrm{~g}$ is provided by coil springs inside the handpiece. The edge of the sleeve is the reference from which measurements are made and the probe has Williams' markings, however actual measurement of the pocket depth is made and transferred automatically to the computer when the foot switch is pressed (Gibbs et al., 1998)

\section{Objective:-}

Clinical attachment level (CAL) remains the gold standard for diagnosis and monitoring of periodontal disease. Aim of the present study is to compare the reproducibility of measurements conducted by two trained examiners obtained by the UNC-15 periodontal probe (1st generation), PDT Sensor probe (2nd generation) and the Florida probe (3rd generation probe).

The aim of this study was also to compare the inter-examiner reproducibility using the UNC-15 Periodontal probe (1st generation), PDT Pressure sensitive probe (2nd generation) and the Florida probe (3rd generation probe) and to obtain a clinical record of probing depth (PD) and CAL with different variables considered. Objective also includes to find out the reliability of three periodontal probes when used by two different examiners. 


\section{Materials and methods:- \\ Inclusion Criteria:-}

Thirty adult male subjects, with an age-range of 25-60 years with more than 14 teeth present in the mouth; having moderate to advanced periodontitis in otherwise systemically healthy patients were included in this study conducted at the Department of Periodontics, Government Dental College and Hospital, Mumbai. Approval was obtained from the Ethical committee Government Dental college and hospital,Mumbai and each participant had to sign a consent form.

Chronic periodontitis patients were selected concurrently with the following criteria, patients with $30 \%$ of sites involved, periodontal pocket depth $\geq 4 \mathrm{~mm}$, attachment loss $\geq 3 \mathrm{~mm}$.

\section{Exclusion criteria:-}

Patients with history of bleeding disorders, or on medication interfering with blood coagulation,pregnant women, patients with history of severe systemic disease e.g. cardiovascular, renal, hepatic or immunologic disorders were excluded in the study.

\section{Procedure:-}

The Gingival index $(<1)$ and Papilla bleeding index $(<1)$ were assessed before probing. Supragingival scaling was performed by ultrasonic scaler four weeks prior to probing procedure.Impressions were taken for both the arches and study models were prepared for the acrylic stents. Thefirst and second probing appointments were scheduled and the duration between the appointments was one week -a time period in which change in attachment level is unlikely to occur. ( Oringer et al.,1997)

Repeated measurements were performed by the same two trained examiners at different appointments. The probes used were the Florida Probe (third generation ), PDT Pressure sensitive probe (second generation), and a conventional UNC-15 manual periodontal probe (first generation). Each pocket was probed thrice with the three probes at 15 minutes interval to avoid excessive bleeding. Measurements were made with the probe tip parallel to the long axis of the tooth at the mid-buccal, mid-lingual /mid -palatal, and as close as possible to the mid interproximal area from the buccal aspect (Marks et al.,1991)

When the pre-set force was achieved, probing depth was recorded to the nearest millimetre. The probe was withdrawn carefully in order to maintain the form of the pocket for the following measurement. Probing depth (PD) and clinical attachment level (CAL) were recorded on three teeth in the same quadrant. Readings were taken from the gingival margin to the bottom of the pocket which represents probing clinical depth. Probing force for pressure probe was calibrated and preset at 15 grams .

Four measurements per tooth were recorded (mesio-buccal, mid-buccal, disto-buccal, and mid-lingual/mid -palatal). All measurements were rounded to the closest $0.5 \mathrm{~mm}$ (up or down), and when the PD measurement was halfway between 2 marks on the probe, the closest millimetre immediately above the mark was recorded .

PD and CAL was recorded by use of UNC 15 periodontal probe, PDT pressure probe and Florida probe in the same sites and same teeth, using custom -made acrylic splint extending on three teeth and with markings on the stent to make probing location consistent .

Before conducting the study, both the examiners were given sufficient time to familiarise themselves with the various probe types. The probing order using different type of probe was randomised, but the same site during the same round, per visit was always evaluated by the identical probe.

\section{Statistical Analysis:-}

The student paired $t$ test was used to compare correlation coefficients between each of the manual probes, $\mathrm{p}$ value less than 0.01 was considered as statistically significant.

The results were analysed statistically by a $t$-test for independent samples (intra-examiner reproducibility) and an analysis of variance (ANOVA) for inter-examiner reproducibility $(\mathrm{p}=0.01)$. By evaluating the results from the first PD (initial) and the repeated PD measurements taken by the two trained examiners, we could calculate the Kappa value and create mathematical measurements for subsequent statistical analyses. Paired $t$ test was performed to find 
out the statistical difference between examiner 1 and 2. Inter examiner reliability was calculated using correlation and alpha values.

Comparison of intra-examiner difference in CAL in case group

Paired Samples Correlations

\begin{tabular}{|c|c|c|c|c|c|c|c|c|c|c|}
\hline \multicolumn{2}{|c|}{ 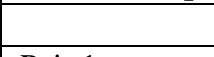 } & \multicolumn{3}{|c|}{ 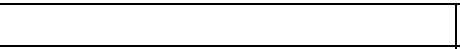 } & \multicolumn{2}{|l|}{$\mathrm{N}$} & \multicolumn{2}{|l|}{ Correlation } & \multicolumn{2}{|c|}{ Significance } \\
\hline \multicolumn{2}{|c|}{ Pair 1} & \multicolumn{3}{|c|}{ UNC1 \& UNC2 } & \multicolumn{2}{|l|}{60} & \multicolumn{2}{|l|}{0.422} & \multicolumn{2}{|c|}{0.001} \\
\hline \multicolumn{2}{|c|}{ Pair 2} & \multicolumn{3}{|c|}{ PDT1 \& PDT 2} & \multicolumn{2}{|l|}{60} & \multicolumn{2}{|l|}{0.303} & \multicolumn{2}{|c|}{0.019} \\
\hline \multicolumn{2}{|c|}{ Pair 3} & \multicolumn{3}{|c|}{ FLORIDA 1 \& FLORIDA 2} & \multicolumn{2}{|l|}{60} & \multicolumn{2}{|l|}{0.495} & \multicolumn{2}{|c|}{.000} \\
\hline & & & \multicolumn{5}{|c|}{ Paired Differences } & $\mathrm{t}$ & df & $\begin{array}{l}\text { Significance } \\
\text { (2-tailed) }\end{array}$ \\
\hline & & & & & & \multicolumn{2}{|c|}{$\begin{array}{l}95 \% \text { Confidence Interval } \\
\text { of the Difference }\end{array}$} & & & \\
\hline & & & Mean & $\begin{array}{l}\text { Std. } \\
\text { Deviation }\end{array}$ & $\begin{array}{l}\text { Std. Error } \\
\text { Mean }\end{array}$ & Lower & Upper & & & \\
\hline $\begin{array}{l}\text { Pair } \\
1\end{array}$ & $\begin{array}{l}\text { UNC1 } \\
\text { UNC2 }\end{array}$ & & -.633 & 1.377 & .178 & -.989 & -.278 & -3.562 & 59 & .001 \\
\hline $\begin{array}{l}\text { Pair } \\
2\end{array}$ & $\begin{array}{l}\text { PDT1 \& } \\
2\end{array}$ & PDT & .033 & 1.449 & .187 & -.341 & .408 & .178 & 59 & .859 \\
\hline $\begin{array}{l}\text { Pair } \\
3\end{array}$ & $\begin{array}{l}\text { FLORII } \\
\text { FLORII }\end{array}$ & $\begin{array}{l}\text { A } 1 \text { - } \\
\text { A } 2\end{array}$ & -.033 & 1.025 & .132 & -.298 & .231 & -.252 & 59 & .802 \\
\hline
\end{tabular}

Comparison of inter -examiner difference in CAL in case group

\section{Paired Samples Correlations}

\begin{tabular}{|l|l|l|l|l|}
\hline & N & Correlation & Sig. \\
\hline Pair 1 & UNCA \& UNCB & 60 & .260 & .045 \\
\hline Pair 2 & PDT1 \& PDT 2 & 60 & .303 & .019 \\
\hline Pair 3 & FLORIDA A \& FLORIDA B & 60 & -.042 & .747 \\
\hline & & & & \\
\hline & & & & \\
\hline
\end{tabular}

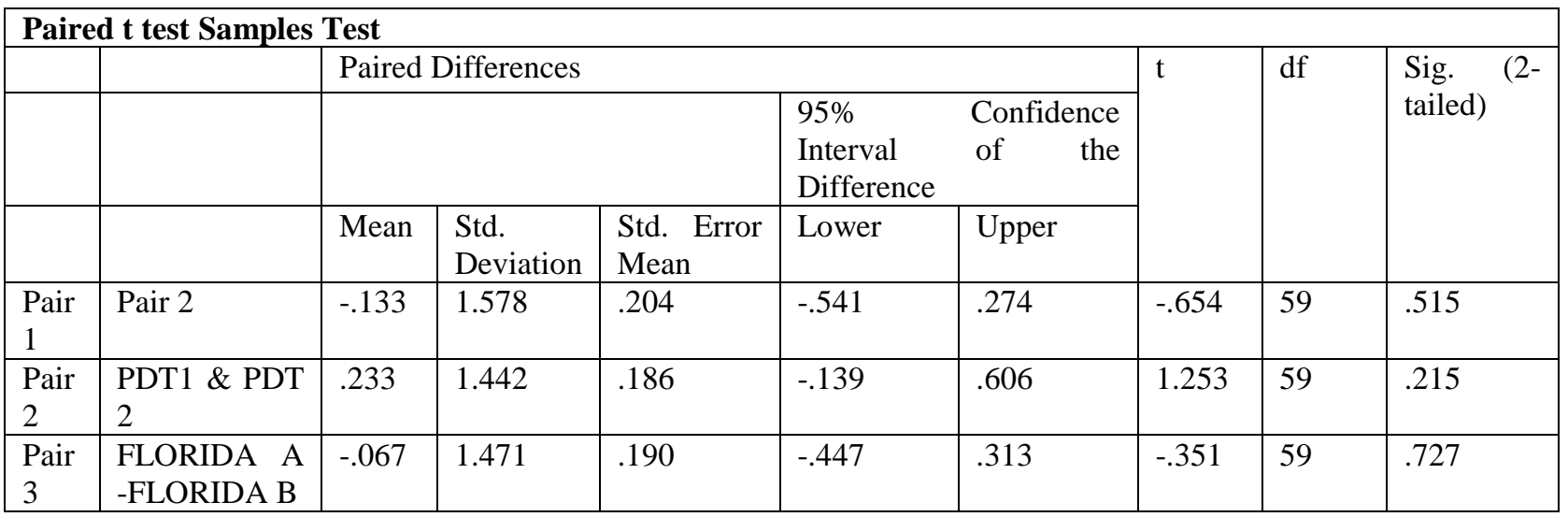

\begin{tabular}{|c|c|c|c|c|c|}
\hline ANOVA & & & & & \\
\hline PROBING DEPT & ESSMENT & & & & \\
\hline & Sum of Squares & $\mathrm{df}$ & Mean Square & $\mathrm{F}$ & Sig. \\
\hline Between Groups & 16.033 & 2 & 8.017 & 20.574 & .000 \\
\hline Within Groups & 68.967 & 177 & .390 & & \\
\hline Total & 85.000 & 179 & & & \\
\hline & & & & & \\
\hline
\end{tabular}


Post Hoc Tests

Multiple Comparisons

PDA (probing depth assessment)

Scheffe

\begin{tabular}{|l|l|l|l|l|l|l|}
\hline (I) PROB & (J) PROB & $\begin{array}{l}\text { Mean Difference } \\
\text { (I-J) }\end{array}$ & Std. Error & Sig. & & $95 \%$ Confidence Interval \\
\cline { 3 - 7 } & & & & & Lower Bound & Upper Bound \\
\hline PDT & FLORIDA & -.117 & .114 & .593 & -.40 & .16 \\
\hline & UNC & $-.683^{*}$ & .114 & .000 & -.96 & -.40 \\
\hline \multirow{2}{*}{ FLORIDA } & PDT & .117 & .114 & .593 & -.16 & .40 \\
\cline { 2 - 6 } & UNC & $-.567^{*}$ & .114 & .000 & -.85 & -.29 \\
\hline \multirow{2}{*}{ UNC } & PDT & $.683^{*}$ & .114 & .000 & .40 & .96 \\
\cline { 2 - 6 } & FLORIDA & $.567^{*}$ & .114 & .000 & .29 & .85 \\
\hline
\end{tabular}

Figure 1 - Recording PD/CAL with UNC-15 probe

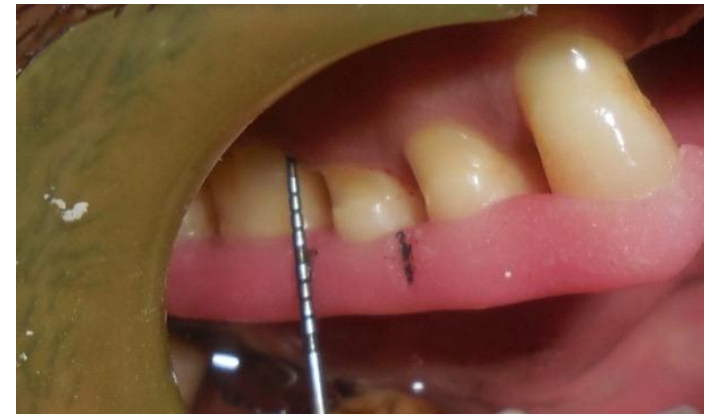

Figure 2- Recording PD/ CAL with PDT pressure sensitive probe

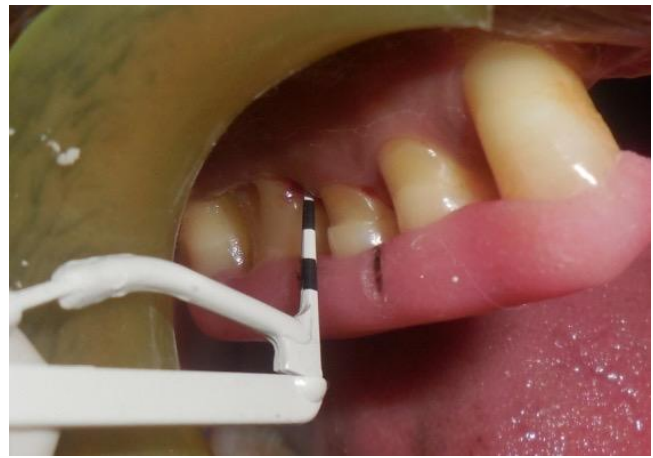

Figure 3- Recording PD/CAL with Florida probe

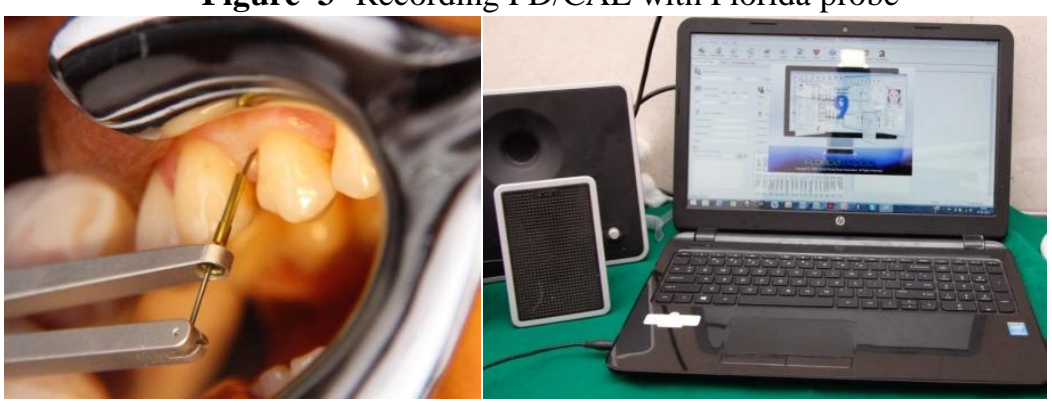


Figure 4- Florida probe calibration

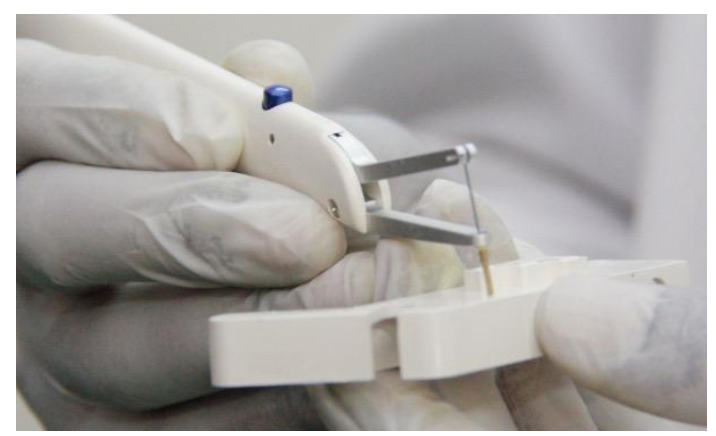

Figure 5- Florida probe Measurements
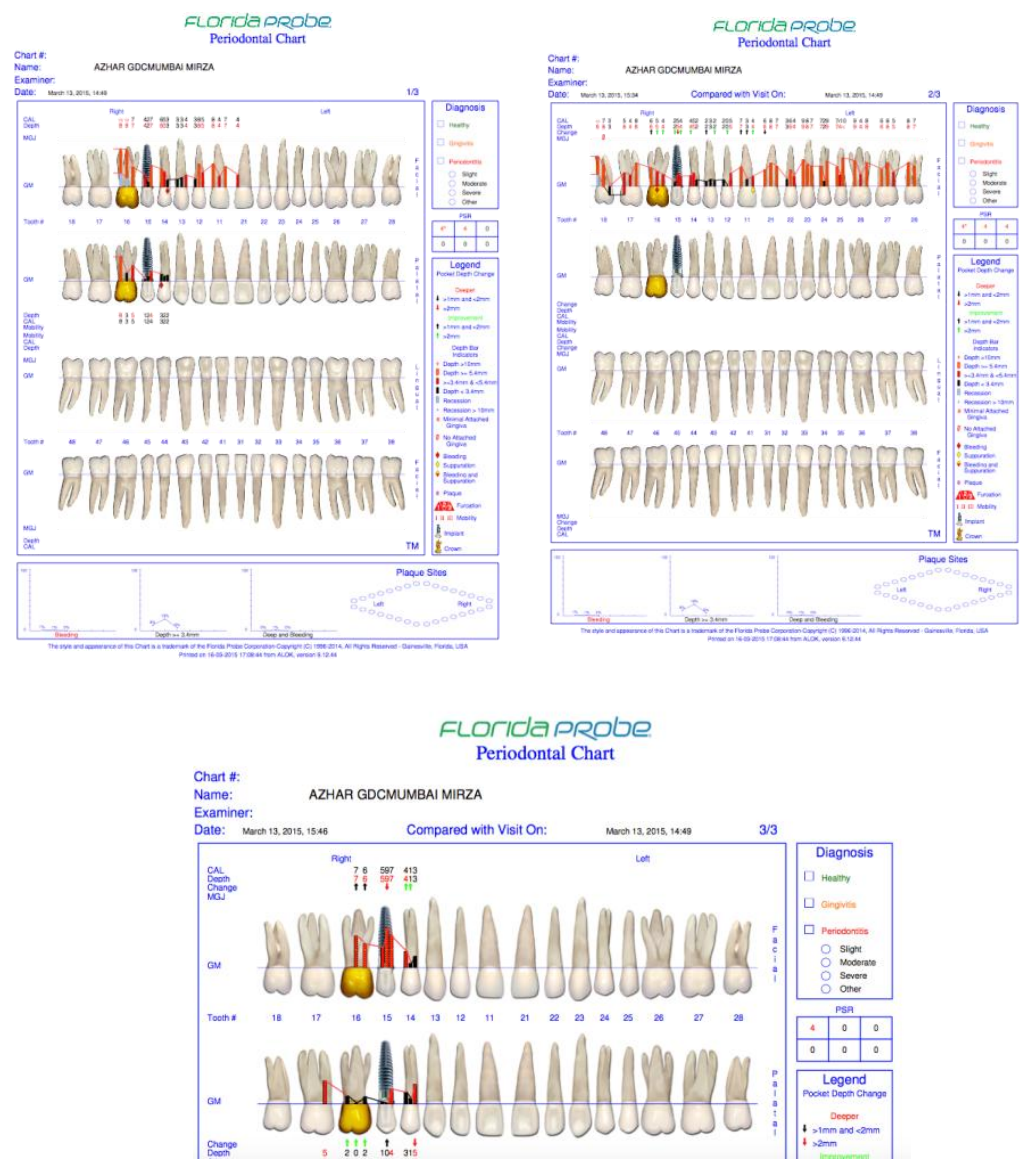

\section{Results:-}

The PD and CAL measurements obtained by UNC 15 probe were higher than PDT pressure sensitive probe and Florida probe but there was no statistical significance in variability of PD and CAL measurements between the conventional and the controlled pressure probe. 
The standard deviations for CAL ranged from $1.59 \mathrm{~mm}$ to $1.89 \mathrm{~mm}(0.30 \mathrm{~mm}$ difference $)$ and from $1.68 \mathrm{~mm}$ to1.96 $\mathrm{mm}$ for PD $(0.28 \mathrm{~mm}$ difference $)$; the Florida Probe produced the lowest standard deviations amongst all the probes.

Reproducibility of measurements by the probes ranged from from 0 to $+1.0 \mathrm{~mm}$ and was $96.49 \%$.Comparison of inter-examiner differences in CAL measurements in all the probes are shown in Tables 3 and 4 respectively.

\section{Discussion:-}

This study evaluated inter-examiner reproducibility between two trained and calibrated examiners as they used a manual probe, pressure sensitive probe and automated probe for clinical recording of PD and CAL in individuals. Periodontal probes are used primarily to detect and measure periodontal pockets and clinical attachment loss. In addition, they are also used to locate calculus, measure gingival recession, measure width of attached gingiva, assess size ofintra-oral lesions, identify tooth and soft tissue anomalies, locate furcation involvements ,determine mucogingival relationships and bleeding tendencies (Wilkins 2005)

Currently, the gold standard for recording changes in periodontal status is longitudinal measurement of CAL from the CEJ or relative attachment level from a fixed reference point (Armitage,1996 and Magnusson,1988). It is of paramount importance that the measuring tools for assessment of periodontal disease activity should be precise and accurate for diagnosis and earlier detection of disease, which in turn, can foster proper and timely treatment as well as long-term periodontal control.

Perry et al in 1994 compared the data from the three generation probes: manual first generation, second generation, and third generation probes.They reported that, when the mean inter-probe PD differences were considered over all sites, the first generation probe produced significantly deeper values than the second generation and third generation probes $(\mathrm{P}<0.0001)$ and that the second generation probe produced values significantly deeper than the third generation probes.In our study too, we found that PD measurements were highest in UNC 15 Probe followed by the PDT Pressure sensitive probe and Florida probe although there was no statistically significant difference in the UNC 15 probe and PDT pressure sensitive probe.

In our study, inter -examiner consistency in measurements of PD and CAL values of Florida probes was found to be better than UNC-15 and PDT pressure sensitive probe. We found that differences in inter-examiner reproducibility were more evident when manual probes were used. This was in agreement to studies (Buduneli et al., 2004,Listgarten 1990 and Osborne et al., 1999) which stated that reproducibility for manual probes was low. Probe angulation, probe tip dimension, and pocket depth will also affect reproducibility (Jeffcoat 1992). Errors in visual assessment, rounding off to the nearest $\mathrm{mm}$, recording errors, variations in probe markings, and patient cooperation must also be considered.

However, studies have shown that the reproducibility of probing over a relatively short period of time may be similar for the conventional and a constant force probe. Wang et al in 1995 concluded that, overall, better reproducibility can be achieved with the conventional than with the electronic probe when compared by tooth surface, tooth type, dental arch or patient.

In our study, both the examiners were trained and experienced postgraduate students in the use of these probes which were being evaluated, due to which the differences between the results obtained by the two examiners were minimal and thus reducing the errors of examiner variability.

Studies done by Preshaw et al in 1997 andKarpinia et al in 2004also included calibrated examiners to minimise the variability error .

Variations in precision of periodontal measurements have also been found to be due to type of teeth (Arthur 1997), sites of measurement(Reddy et al., 1997), probing force (Garnick et al 2000), probe placement and angulation (Persson 1991), visual and tactile difficulty in detecting the CEJ (Watts 1987) and erroneous recording of measurements. 
In our study,each pocket was probed thrice with the three probes at 15 minutes interval to avoid excessive bleeding.The 15-min interval was used based on studies which reported that allowing a 15-minute interval between initial and repeated probes in the clinical environment reduced the risk of bleeding (Van der Velden et al.,1980). The second-generation instruments are pressure sensitive, allowing for improved standardisation of probing pressure. Scientific literature that demonstrated probing pressure should be standardised and not exceed $0.2 \mathrm{~N} / \mathrm{mm}^{2}$ led to the development of these probes.Second- generation probes can be used in general dental practices, as well as periodontal practices, and do not require computerisation in the operatory. (Arthur 1997)

In our study, although the UNC-15 the first generation probe produced deeper PD and CAL values than the PDT pressure sensitive probe and Florida probe, there was no statistical difference between the measurements in PD and CAL between UNC 15 probe and PDT pressure sensitive probe .This was similar to results obtained ( Rocha et al., 2003) which stated that there is no great variability of PD between the conventional and the controlled pressure probe.

In this study, the Florida probe was found to be the greatest potential for accuracy, and inter-examiner consistency and reliability in detecting clinical attachment level in compared to PDT pressure sensitive probe and UNC-15. This is in accordance with studies which have shown that Florida probe system has given superior results to manual UNC- 15 periodontal probe in measuring the pocket depth and CAL accurately (R Deepa et al ., 2012) By automated measurements, direct computerised data collection, visual observational and transcribing errors are eliminated, which is usually seen with non automated probes (Watts 1995).

The software of the electronic periodontal probe also permits a risk evaluation for periodontal diseases, which is especially important for the medical investigator, offering him a global view of the patient, but also for the patient himself, who can be directly informed and motivated on his own periodontal status (Alexandra 2014)

\section{Conclusion:-}

There is no great variability of PD and CAL between the conventional and the controlled pressure probe. PDT probe and Florida probe have been shown to be more accurate and were found to be more consistent which were reproducible by two independent examiners.

Within the limitations of our study, Florida probe has proved to have the greatest potential for accuracy and reliability for measurements of PD and CAL than PDT Pressure sensitive probe and manual probe (UNC-15), indicating that automated probes are better as far as accuracy, consistency, and reliability are concerned for measurements of attachment levels.

\section{Acknowledgments:-}

The authors wish to thank Dr Shine Simon ,Post graduate student,Department of Periodontology, Government Dental college and hospital,Mumbai,for documentation and photography.We are extremely thankful to Mr. Alok Vashista for providing Florida probe and other relevant information in time.

\section{References:-}

1. Alexandra Martu,Cristala Nitescu.(2014): Comparative study on the efficiency of periodontal probing with electronic periodontal probe versus conventional periodontal probe.International Journal of Medical Dentistry .volume 4 issue 4 October/December.

2. Armitage. (1996): Periodontal disease: Diagnosis. Ann Periodontol; 1:54-83

3. Arthur F.H . (1997): Periodontal probing Crit. Rev. Oral Biol. Med; 8:336- 356.

4. Buduneli E, Aksoy O, Köse T, Atilla G.(2004): Accuracy and reproducibility of two manual periodontal probes; an in- vitro study. J Clin Periodontol ;Oct;31(10):815-9

5. Garnick JJ, Silverstein L.(2000): Periodontal Probing: probe tip diameter. J Periodontol ; 71:96103.

6. Gibbs CH, Hirschfeld IW, Lee JG, Low SB, Magnusson I,(1988): Description and clinical evaluation of a new computerized periodontal probe-the Florida Probe. J Clin Periodontol ;15:137-44.

7. Grossi SG, Dunford et al.(1996): Sources of error for periodontal probing measurements. J Periodont Res ;31:330336. 
8. Hunter FM, Martin ND, Godfrey K.(1990): Development of a disposable pressure sensitive periodontal probe and comparison of probing forces. J Dent Res ;69:143.

9. Jeffcoat MK.(1992):Radiographic methods for the detection of progressive alveolar bone loss. J Periodontol ; 63:367.

10. Karpinia K, Magnusson I, Gibbs C, Yang MC.(2004): Accuracy of probing attachment levels using a CEJ Probe versus traditional probes. J Clin Periodontol ;31:173-6.

11. Listgarten MA. (1980):Periodontal probing: what does it mean? J Clin Periodontol. Jun;7(3):165-76.

12. Magnusson I, Clark WB, Marks RG, Gibbs CH, Manouchehr-Pour M, Low SB.(1988): Attachment level measurements with a constant force electronic probe. J Clin Periodontol ;15:185-8

13. Marks RG, Low SB, Taylor M, Baggs R, Magnusson I, Clark WB. (1991): Reproducibility of attachment level measurements with two models of the Florida Probe. J Clin Periodontol ;18:780-4.

14. Newman T, Klokkevold C. (2006): Clinical Periodontology. 10th ed. Philadelphia: WB Saunders

15. Oringer RJ, Fiorellini JP, Koch GG, Sharp TJ, Nevins ML, Davis GH( 1997): Comparison of manual and automated probing in an untreated periodontitis population. J Periodontol ;68:1156-62.

16. Osborn J, Stoltenberg JL, Huso BA, Aeppli DM, Pihlstrom BL.(1990): Comparison of measurement variability using a standard and constant force periodontal probe. J Periodontol ;61:496-503.

17. Philstrom BL (1992): Measurement of attachment level in clinical trials: Probing methods. J Periodontol ;63:1072- 1077

18. Parakkal PF.(1979): Proceedings of the workshop on quantitative evaluation of periodontal disease by physical measurement techniques. J Dent Res; 58: 547-53.

19. Perry DA, Taggart EJ, Leung A, Newbrun E.(1994): Comparison of a conventional probe with electronic and manual pressure-regulated probes. J Periodontol ;65:908-913 .

20. Persson GR.(1991): Effects of line angle versus mid proximal periodontal probing measurements on prevalence estimates of periodontal disease. J Periodontol Res;26:527529.

21. Preshaw PM, Kupp L, Hefti AF, Mariotti A. (1997): Measurement of clinical attachment levels using a constant-force periodontal probe modified to detect the cement-enamel junction. J Clin Periodontol ;26:434-40.

22. Philstrom BL (1992): Measurement of attachment level in clinical trials: Probing methods. IPeriodontol ;63:1072- 1077.

23. R Deepa, Shobha Prakash.(2012): Accuracy of probing attachment levels using a new computerized cementoenamel junction probe. Journal of Indian Society of Periodontology; - Vol 16, Issue 1, Jan-Mar.

24. Reddy MS, Palcanis KG, Geurs NC. (1997):A comparison of manual and controlled force attachment level measurements. J Clin Periodontol ;24:920926

25. Roberto Andrade,Manuel Espinoza, Elena Maria Gómez, José Rolando Espinoza,Elizabeth Cruz . (2012): Intraand inter-examiner reproducibility of manual probing depth Braz Oral Res; Jan-Feb;26(1):57-63

26. Rocha, E. F. et al. (2003):Variability of the probing depth between conventional and controlled-force periodontal probes. Salusvita, Bauru; v. 22, n. 2, p. 219-227.

27. Van der Velden, U., de Vries, I.H.(1980): The influence of probing force on the reproducibility of pocket depth measurements. J. Clin. Periodontol; 7, 414-420 .

28. Wang SF, Leknes k, Zimmerman GJ, et al.(1995): Reproducibility of periodontal probing using a conventional manual and an automated force controlled electronic probe. J Periodontol ;66:3846

29. Watts TP. (1995) Periodontal disease activity: A development strategy for its investigation by means of accurate three dimensional clinical measurement. J Clin Periodontol;22:201-7.

30. Wilkins Esther (2005): Clinical Practice of the Dental Hygienist 9th ed. Philadelphia, PA: Lippincott Williams and Wilkins; 222- 245. 